\title{
Scalar fields, energy conditions and traversable wormholes
}

\author{
Carlos Barceló $\dagger$ and Matt Visserł \\ Physics Department, Washington University, Saint Louis, MO 63130-4899, USA \\ E-mail: carlos@hbar.wustl.edu and visser@kiwi.wustl.edu
}

Received 8 March 2000, in final form 20 July 2000

\begin{abstract}
We describe the different possibilities that a simple and apparently quite harmless classical scalar field theory provides to violate the energy conditions. We demonstrate that a nonminimally coupled scalar field with a positive curvature coupling $\xi>0$ can easily violate all the standard energy conditions, up to and including the averaged null energy condition (ANEC). Indeed, this violation of the ANEC suggests the possible existence of traversable wormholes supported by non-minimally coupled scalars. To investigate this possibility we derive the classical solutions for gravity plus a general (arbitrary $\xi$ ) massless non-minimally coupled scalar field, restricting attention to the static and spherically symmetric configurations. Among these classical solutions we find an entire branch of traversable wormholes for every $\xi>0$. (This includes and generalizes the case of conformal coupling $\xi=\frac{1}{6}$ we considered in 1999 Phys. Lett. B 466 127-34.) For these traversable wormholes to exist we demonstrate that the scalar field must reach trans-Planckian values somewhere in the geometry. We discuss how this can be accommodated within the current state of the art regarding scalar fields in modern theoretical physics. We emphasize that these scalar field theories, and the traversable wormhole solutions we derive, are compatible with all known experimental constraints from both particle physics and gravity.
\end{abstract}

PACS numbers: 0460D, 0462, 9880H

\section{Introduction}

It is often (mistakenly) believed that every kind of matter, on scales for which we do not need to consider its quantum features, has an energy density that is everywhere positive. (In fact, we could think of this property as defining what we understand by classical matter.) This is more precisely stated by saying that every type of classical matter satisfies the energy conditions of general relativity [1]. There are several (pointwise) energy conditions requiring that various linear combinations of the components of the energy-momentum tensor of matter have positive values. (Or at the very least, non-negative values.) Among them, we will be particularly interested in the null energy condition (NEC) because it is the weakest one: if the NEC is violated all pointwise energy conditions would be violated [2].

Now, if we assume that the energy conditions are satisfied by every kind of classical matter, general relativity leads to many powerful classical theorems. The singularity theorems [1], the positive mass theorem [3], the superluminal censorship theorem [4-6], the topological censorship theorem [7], certain types of no-hair theorem [8] and various constraints on black hole surface gravity [9], all make use of some type of energy condition. As an illustration of the importance of these results, the conclusion regarding the inevitability of the appearance of

$\dagger$ Homepage: http://www.physics.wustl.edu/ carlos

† Homepage: http://www.physics.wustl.edu/ visser 
singularities [1], has been (for the last 30 years) one of the central pillars from which many investigations in general relativity start. (Additional powerful technical assumptions are also needed for the singularity theorems to apply; see [10] for a critical review.) In this regard, the alleged impossibility of the existence of traversable wormholes connecting different spatial regions of the universe $[7,11]$, a topic in which we will be particularly interested in this paper, could be seen as a complementary way of phrasing that conclusion (of course, one should not carry this parallelism too far).

Assuming the positivity of the energy density implies that spacetime geometries containing traversable wormholes $\dagger$ are ruled out of the classical realm. Specifically, the topological censorship theorem [7] states that if the averaged null energy (ANEC; the NEC averaged over a complete null geodesic) is satisfied, then there cannot be any topological obstruction (e.g. a wormhole) inside any asymptotically flat spacetime. In agreement with this result, a purely local analysis by David Hochberg and one of the present authors [12] shows that the violation of NEC on or near the throat of a traversable wormhole is a generic property of these objects. For this reason most of the investigations regarding traversable wormholes tend to view these objects as semiclassical in nature, using the expectation value of the quantum operator associated with the energy-momentum tensor as the source of gravity [13].

However, it is easy to demonstrate that an extremely simple and apparently quite innocuous classical field theory, a scalar field non-minimally coupled to gravity (that is, with a nonvanishing coupling to the scalar curvature), can violate the NEC and even the ANEC [14-16]. As we will see, some of the other energy conditions can be violated even by minimally coupled scalar fields. There exist other classical systems that exhibit NEC violations, such as BransDicke theory [17-20], higher-derivative gravity [21] or Gauss-Bonnet theory [22], but they are all based on modifications of general relativity at high energies. It is the simplicity of the scalar field theory that particularly attracted our attention.

In a previous paper [15] we analysed a massless scalar field conformally coupled to gravity (that is, we used the special value $\xi=\frac{1}{6}$ ) and found that among the classical solutions for the system there exists an entire branch of traversable wormholes. The case of conformal coupling has many interesting features and, in fact, it seems the most natural behaviour for a scalar field at low energies $[15,16]$. However, in this paper we want to point out that assuming conformal coupling is not the critical issue, and that it is only the fact of non-minimality (more precisely, positive curvature coupling $\xi>0$ ) that is important for traversable wormhole solutions to exist. We have found general expressions for the classical solutions of gravity plus a massless non-minimally coupled scalar field. In particular, we have found a sub-class of traversable wormhole solutions for the entire range $\xi>0$. In our solutions, apart from the geometry, there is also a scalar field that modifies the effective Newton constant. We will not address here the possible effects of this scalar field on the journey of any hypothetical traveller trying to cross the wormhole throat.

In the next section we will review the possibilities that scalar fields offer to violate the different energy conditions. (That scalar fields might potentially cause problems in this regard was first noted at least 25 years ago [23], and is an issue that has periodically come in and out of focus since then [16].) Then we will obtain and describe the classical solutions for gravity plus a non-minimally coupled massless scalar field, restricting to static and spherically symmetric configurations. Among these solutions there are an assortment of naked singularities but we

$\dagger$ The term 'traversable wormhole' was adopted by Morris and Thorne [11] to describe a class of Lorentzian geometries connecting two asymptotically flat regions of spacetime, in a manner suitable for a signal or particle to pass through in both directions. By the definition of traversability it should be possible to travel from one asymptotic region to the other without encountering either horizons or naked singularities. In this paper we use this term exclusively from this geometrical point of view. 
also find an entire branch of traversable wormhole solutions for curvature coupling $\xi>0$. We will leave it to section 4 to analyse these traversable wormhole geometries in detail. Finally, in section 5 we will discuss the key features of these solutions and the plausibility with which they might actually be produced in nature, based on the role of scalar fields in modern theoretical physics and the totality of the experimental constraints arising from both particle physics and gravity physics.

\section{Scalar fields and energy conditions}

When a classical scalar field acts as a source of gravity, many of the energy conditions can be violated depending on the form of the scalar potential and the value of the curvature coupling.

\subsection{Effective stress-energy tensor}

The Einstein equations $\kappa G_{\mu \nu}=T_{\mu \nu}$ relate the geometry-dependent Einstein tensor $G_{\mu \nu}$ to the energy-momentum tensor for the matter field $T_{\mu \nu}$. The symbol $\kappa$ represents essentially the inverse of Newton's constant, $\kappa=1 /\left(8 \pi G_{\mathrm{N}}\right)$. These equations can be obtained by varying the Einstein-Hilbert action which, for a generically coupled scalar field, reads

$\mathcal{S}=\frac{1}{2} \int \mathrm{d}^{4} x \sqrt{-g} \kappa R+\int \mathrm{d}^{4} x \sqrt{-g}\left(-\frac{1}{2} g^{\mu \nu} \partial_{\mu} \phi_{\xi} \partial_{\nu} \phi_{\xi}-V\left(\phi_{\xi}\right)-\frac{1}{2} \xi R \phi_{\xi}^{2}\right)$.

Then, the scalar field energy-momentum tensor has the form [14]

$$
\begin{aligned}
{\left[T\left(\phi_{\xi}\right)\right]_{\mu \nu}=} & \nabla_{\mu} \phi_{\xi} \nabla_{\nu} \phi_{\xi}-\frac{1}{2} g_{\mu \nu}\left(\nabla \phi_{\xi}\right)^{2}-g_{\mu \nu} V\left(\phi_{\xi}\right) \\
& +\xi\left[G_{\mu \nu} \phi_{\xi}^{2}-2 \nabla_{\mu}\left(\phi_{\xi} \nabla_{\nu} \phi_{\xi}\right)+2 g_{\mu \nu} \nabla^{\lambda}\left(\phi_{\xi} \nabla_{\lambda} \phi_{\xi}\right)\right] .
\end{aligned}
$$

This energy-momentum tensor has a term that depends algebraically on the Einstein tensor. By grouping all the dependence on $G_{\mu \nu}$ on the left-hand side of the Einstein equations we can rewrite them, alternatively, by using an effective energy-momentum tensor

$$
\begin{aligned}
{\left[T^{\mathrm{eff}}\left(\phi_{\xi}\right)\right]_{\mu \nu}=} & \frac{\kappa}{\kappa-\xi \phi_{\xi}^{2}}\left[\nabla_{\mu} \phi_{\xi} \nabla_{\nu} \phi_{\xi}-\frac{1}{2} g_{\mu \nu}\left(\nabla \phi_{\xi}\right)^{2}-g_{\mu \nu} V\left(\phi_{\xi}\right)\right. \\
& \left.-\xi\left[2 \nabla_{\mu}\left(\phi_{\xi} \nabla_{\nu} \phi_{\xi}\right)-2 g_{\mu \nu} \nabla^{\lambda}\left(\phi_{\xi} \nabla_{\lambda} \phi_{\xi}\right)\right]\right] .
\end{aligned}
$$

This is the relevant expression for the analysis of the different energy conditions: since the Einstein equations now read $\kappa G_{\mu \nu}=\left[T^{\mathrm{eff}}\left(\phi_{\xi}\right)\right]_{\mu \nu}$, a constraint on this effective stress-energy tensor is translated directly into a constraint on the spacetime curvature, and it is ultimately constraints on the spacetime curvature that lead to singularity theorems and the like.

\subsection{Pointwise energy conditions}

Now let $v^{\mu}$ be a properly normalized timelike vector, $\left(v^{2}=-1\right)$, and, for convenience, let it be locally extended to a geodesic vector field, so that $v^{\mu} \nabla_{\mu} v^{\nu}=0$. If $x^{\mu}(\tau)$ denotes a timelike geodesic with tangent vector $v^{\mu}=\mathrm{d} x^{\mu} / \mathrm{d} \tau$ we have $v^{\mu} \nabla_{\mu} \phi=\mathrm{d} \phi / \mathrm{d} \tau \equiv \phi^{\prime}$. We can now express the strong energy condition (SEC) as

$$
\begin{aligned}
R_{\mu \nu} v^{\mu} v^{\nu} & =\frac{1}{\kappa}\left(\left[T^{\mathrm{eff}}\left(\phi_{\xi}\right)\right]_{\mu \nu}-\frac{1}{2} g_{\mu \nu}\left[T^{\mathrm{eff}}\left(\phi_{\xi}\right)\right]\right) v^{\mu} v^{\nu} \\
& =\frac{1}{\kappa-\xi \phi_{\xi}^{2}}\left[\left(\phi_{\xi}^{\prime}\right)^{2}-V\left(\phi_{\xi}\right)-\xi\left[\left(\phi_{\xi}^{2}\right)^{\prime \prime}-\nabla^{\mu}\left(\phi_{\xi} \nabla_{\mu} \phi_{\xi}\right)\right]\right] \geqslant 0,
\end{aligned}
$$


where $R_{\mu \nu}$ is the Ricci tensor of the geometry. It is easy to see that even in the minimally coupled case the SEC can be violated for positive values of the scalar potential such as a mass term or a positive cosmological constant. Every cosmological inflationary process violates the SEC $[16,24]$. We could say that violation of the SEC is a generic property of scalar fields. In fact, it is so easy to violate SEC in many situations that it has almost become abandoned [16] as a reasonable restriction on the properties of matter.

With the same definitions, the weak energy condition (WEC) reads

$$
\begin{aligned}
G_{\mu \nu} v^{\mu} v^{v}=\frac{1}{\kappa} & {\left[T^{\mathrm{eff}}\left(\phi_{\xi}\right)\right]_{\mu \nu} v^{\mu} v^{v}=\frac{1}{\kappa-\xi \phi_{\xi}^{2}} } \\
& \times\left[(1-2 \xi)\left(\phi_{\xi}^{\prime}\right)^{2}+\frac{1}{2}\left(\nabla \phi_{\xi}\right)^{2}+V\left(\phi_{\xi}\right)-\xi\left[\left(\phi_{\xi}^{2}\right)^{\prime \prime}+2 \nabla^{\mu}\left(\phi_{\xi} \nabla_{\mu} \phi_{\xi}\right)\right]\right] \geqslant 0 .
\end{aligned}
$$

In the minimally coupled case $(\xi=0)$ it is generically satisfied. Only a large negative potential, for example, a negative cosmological constant, could provide a violation of the WEC. Although recent observations suggest a probable positive value for the effective cosmological constant, a possible negative value cannot be ruled out on theoretical grounds. In the non-minimal case there are various terms that can be negative depending on the situation, so the WEC can be violated in various ways.

Finally, let us now analyse the NEC. It is the weakest pointwise energy condition, that is, when it is violated the WEC and SEC are violated too. Let $k^{\mu}$ be a null vector tangent to the null geodesic $x^{\mu}(\lambda)$, with $\lambda$ some affine parameter. In an analogous way as with the previous energy conditions, we arrive at the following expression for the NEC:

$$
G_{\mu \nu} k^{\mu} k^{\nu}=\frac{1}{\kappa}\left[T^{\mathrm{eff}}\left(\phi_{\xi}\right)\right]_{\mu \nu} k^{\mu} k^{\nu}=\frac{1}{\kappa-\xi \phi_{\xi}^{2}}\left[\phi_{\xi}^{\prime 2}-\xi\left(\phi_{\xi}^{2}\right)^{\prime \prime}\right] \geqslant 0 .
$$

This condition is clearly satisfied by minimally coupled scalars. However, for $\xi \neq 0$ it can be violated in a number of ways: for $\xi<0$ any local minimum of $\phi_{\xi}^{2}$ violates the NEC, while for $\xi>0$ and $\left|\phi_{\xi}\right|$ small (meaning $\left|\phi_{\xi}\right|<(\kappa / \xi)^{1 / 2}$ ), any local maximum of $\phi_{\xi}^{2}$ violates the NEC. Finally, for $\xi>0$ and $\left|\phi_{\xi}\right|$ large (meaning $\left|\phi_{\xi}\right|>(\kappa / \xi)^{1 / 2}$, roughly corresponding to super-Planckian values for the scalar field), any local minimum of $\phi_{\xi}^{2}$ violates the NEC.

At this point it is worth noting that, because our analysis is completely classical, it is a priori conceivable that averaged versions of the energy conditions (averaged over a geodesic) could in principle be as easily violated as their pointwise counterparts. In particular, ANEC violations, critical for traversable wormhole configurations to be able to exist, could in principle be as easy to find as NEC violations, and it is to exploring this possibility that we now turn.

\subsection{Averaged energy conditions-ANEC}

Suppose we take a segment of a null geodesic and consider the ANEC integral

$$
\mathcal{I}\left(\lambda_{1}, \lambda_{2}\right)=\int_{\lambda_{1}}^{\lambda_{2}}\left[T^{\mathrm{eff}}\left(\phi_{\xi}\right)\right]_{\mu \nu} k^{\mu} k^{\nu} \mathrm{d} \lambda
$$

Then

$$
\mathcal{I}\left(\lambda_{1}, \lambda_{2}\right)=\int_{\lambda_{1}}^{\lambda_{2}} \frac{\kappa}{\kappa-\xi \phi_{\xi}^{2}}\left\{\left(\frac{\mathrm{d} \phi_{\xi}}{\mathrm{d} \lambda}\right)^{2}-2 \xi \frac{\mathrm{d}}{\mathrm{d} \lambda}\left(\phi_{\xi} \frac{\mathrm{d} \phi_{\xi}}{\mathrm{d} \lambda}\right)\right\} \mathrm{d} \lambda .
$$


Integrate by parts

$\mathcal{I}\left(\lambda_{1}, \lambda_{2}\right)=\int_{\lambda_{1}}^{\lambda_{2}} \frac{\kappa}{\kappa-\xi \phi_{\xi}^{2}}\left\{\left(\frac{\mathrm{d} \phi_{\xi}}{\mathrm{d} \lambda}\right)^{2}+\frac{4 \xi^{2} \phi_{\xi}^{2}\left(\mathrm{~d} \phi_{\xi} / \mathrm{d} \lambda\right)^{2}}{\kappa-\xi \phi_{\xi}^{2}}\right\} \mathrm{d} \lambda-\left.\left\{\frac{2 \xi \kappa}{\kappa-\xi \phi_{\xi}^{2}}\left(\phi_{\xi} \frac{\mathrm{d} \phi_{\xi}}{\mathrm{d} \lambda}\right)\right\}\right|_{\lambda_{1}} ^{\lambda_{2}}$.

Now assemble the pieces:

$\mathcal{I}\left(\lambda_{1}, \lambda_{2}\right)=\int_{\lambda_{1}}^{\lambda_{2}} \frac{\kappa\left[\kappa-\xi(1-4 \xi) \phi_{\xi}^{2}\right]}{\left(\kappa-\xi \phi_{\xi}^{2}\right)^{2}}\left(\frac{\mathrm{d} \phi_{\xi}}{\mathrm{d} \lambda}\right)^{2} \mathrm{~d} \lambda-\left.\left\{\frac{2 \xi \kappa}{\kappa-\xi \phi_{\xi}^{2}}\left(\phi_{\xi} \frac{\mathrm{d} \phi_{\xi}}{\mathrm{d} \lambda}\right)\right\}\right|_{\lambda_{1}} ^{\lambda_{2}}$.

Discarding the boundary terms is an issue fraught with subtlety. We start by considering a complete null geodesic and assuming sufficiently smooth asymptotic behaviour. Then the boundary terms from asymptotic infinity can be neglected, and the only potential problems with the boundary terms come from the places $\lambda_{i}$ where $\kappa=\xi \phi_{\xi}\left(\lambda_{i}\right)^{2}$. We obtain

$\mathcal{I}(-\infty,+\infty)=\oint \frac{\kappa\left[\kappa-\xi(1-4 \xi) \phi_{\xi}^{2}\right]}{\left(\kappa-\xi \phi_{\xi}^{2}\right)^{2}}\left(\frac{\mathrm{d} \phi_{\xi}}{\mathrm{d} \lambda}\right)^{2} \mathrm{~d} \lambda+\left.\sum_{i}\left\{\frac{2 \xi \kappa}{\kappa-\xi \phi_{\xi}^{2}}\left(\phi_{\xi} \frac{\mathrm{d} \phi_{\xi}}{\mathrm{d} \lambda}\right)\right\}\right|_{\lambda_{i}^{+}} ^{\lambda_{i}^{-}}$.

(a) If $\xi<0$ then there are no places on the geodesic where $\kappa=\xi\left[\phi_{\xi}(\lambda)\right]^{2}$, so the boundary terms represent an empty set. However, if $\xi<0$ the integrand in the above formula is itself guaranteed to be positive so the ANEC is satisfied.

(b) If $\xi>0$, but we have $\left(\phi_{\xi}\right)^{2}<\kappa / \xi$, then again there are no places on the geodesic where $\kappa=\xi\left[\phi_{\xi}(\lambda)\right]^{2}$. Furthermore, the integrand appearing above is again positive and the ANEC is satisfied.

(c) Finally, if $\xi>0$, and we have at least some places where $\left(\phi_{\xi}\right)^{2}>\kappa / \xi$, then there are by definition places on the geodesic where $\kappa=\xi\left[\phi_{\xi}(\lambda)\right]^{2}$. The boundary terms can no longer be neglected and potentially can contribute-typically making negative and infinite contributions to the ANEC integral. Furthermore, the integrand appearing above is no longer guaranteed to be positive. (If $\xi \in\left(0, \frac{1}{4}\right)$ then it is possible to have $\left(\phi_{\xi}\right)^{2}>\kappa /[\xi(1-4 \xi)]$ and so make the integrand negative.) In short, there is definitely the possibility of ANEC violations under these conditions, and in the exact solutions we investigate below we shall see that for some of these exact solutions the ANEC is certainly violated.

Thus we have seen that a rather simple and apparently quite harmless scalar field theory can in many cases violate all the energy conditions. Violating all the pointwise energy conditions is particularly simple, and violating the averaged energy conditions, though more difficult, is still generically possible. In the next section we shall exhibit some specific examples of this phenomenon in the form of exact solutions to the coupled Einstein-scalar-field equations. In the final section we will discuss the extent to which these exact solutions are realistic: we shall discuss the generic role played by scalar fields in modern theoretical physics, and the experimental/observational limits on their existence and behaviour in order to address the physical plausibility of these energy condition violations.

\section{Non-minimal classical solutions}

As we have just seen, a non-minimally coupled scalar field can violate, in some circumstances, the NEC and even the ANEC. This opens up the possibility of finding some traversable 
wormholes among the many geometries that can be supported by a classical non-minimally coupled scalar field. That this is, in fact, the case for a massless conformally coupled scalar field was shown in [15]. In the present paper, we will see that even for nonconformal coupling (curvature coupling different from $\frac{1}{6}$ ), but positive, we can also find traversable wormholes. In this section we will obtain the classical solutions for gravity plus a generic non-minimally coupled scalar field. For simplicity, we will restrict to the spherically symmetric and static configuration and will take the scalar potential $V(\phi)$ as equal to zero. (To add a particle mass to the scalar field complicates the equations sufficiently to preclude the possibility of analytic results.)

\subsection{Some 'trivial' solutions}

The first thing that we realize is that for any spatially constant value of the scalar field, $\phi_{\xi}=C$, the Einstein equations reduce to $\kappa G_{\mu \nu}=\xi G_{\mu \nu} C^{2}$. By looking also at the scalar field equation $\left(\nabla^{2} \phi_{\xi}=\xi R \phi_{\xi}\right) \Rightarrow \xi R \phi_{\xi}=0$, where $R$ is the Ricci scalar curvature, we can straightforwardly find a variety of trivial solutions for this system. For (a) $\xi>0$ and $C \neq \pm \sqrt{\kappa / \xi}$, or (b) $\xi<0$ and any value of $C$, we find that the ordinary vacuum Einstein solutions also solve the coupled Einstein-scalar equations. As here we are restricted to spherically symmetric and static configurations without a cosmological constant, the solutions that show up are the Schwarzschild and anti-Schwarzschild geometries $(M<0$ represents a perfectly good solution to the Einstein field equations, normally the negative mass Schwarzschild geometry is excluded by hand, here it is best to keep it for the time being as an aid in classifying the total solution space).

More surprising is that for $\xi>0$ and $C= \pm \sqrt{\kappa / \xi}$ every Ricci-scalar-flat geometry is a solution. (That is, any geometry satisfying the condition $R=0$ is a solution of the coupled Einstein-scalar equations.) The condition $R=0$ is characteristic of geometries supported by conformally invariant matter. In particular, the geometries that appear in the solutions for the $\xi=\frac{1}{6}$ case all satisfy $R=0$. These geometries were found in Froyland [25] and also in [15], and will be re-obtained later on this paper as particular cases. Here we want to point out that these geometries are not only associated with the conformal coupling but they appear quite generally, for arbitrary $\xi>0$, provided only $\phi_{\xi}=C \equiv \pm \sqrt{\kappa / \xi}$.

\subsection{Solution-generating technique}

To obtain additional (non-trivial) solutions for a scalar field non-minimally coupled to gravity we will use a 'solution-generating technique' that relies on knowledge of the solutions for the minimally coupled case $[26,27]$. The classical solutions for a massless scalar field minimally coupled to gravity are very well known. They have been discovered and rediscovered several times in different coordinate systems (see the papers by Fisher [28], Janis et al [29], Wyman [30], and Cavaglia and De Alfaro [31]). They can be expressed [32] as

$$
\begin{aligned}
& \mathrm{d} s_{m}^{2}=-\left(1-\frac{2 \eta}{r}\right)^{\cos \chi} \mathrm{d} t^{2}+\left(1-\frac{2 \eta}{r}\right)^{-\cos \chi} \mathrm{d} r^{2}+\left(1-\frac{2 \eta}{r}\right)^{1-\cos \chi} r^{2}\left(\mathrm{~d} \theta^{2}+\sin ^{2} \theta \mathrm{d} \Phi^{2}\right) \\
& \phi_{m}=\sqrt{\frac{\kappa}{2}} \sin \chi \ln \left(1-\frac{2 \eta}{r}\right) .
\end{aligned}
$$

The same geometry, which has an obvious symmetry under $\chi \rightarrow-\chi$, can exist with a field configuration $\phi_{m}$ or the reversed sign configuration $-\phi_{m}$. Less obvious is that by making 
a coordinate transformation $r \rightarrow \tilde{r}=r-2 \eta$, one uncovers an additional symmetry under $\{\eta, \chi\} \rightarrow\{-\eta, \chi+\pi\}$, (with $\phi_{m} \rightarrow+\phi_{m}$ ). The key to this symmetry is to realize that

$$
\left(1-\frac{2 \eta}{r}\right)=\left(1+\frac{2 \eta}{\tilde{r}}\right)^{-1}
$$

In view of these symmetries one can without loss of generality take $\eta \geqslant 0$ and $\chi \in[0, \pi]$ remembering the overall two possible signs for the scalar field. Similar symmetries will be encountered for non-minimally coupled scalars.

The Lagrangian for which these 'minimal' solutions are extrema is

$$
S_{m}=\frac{1}{2} \int \mathrm{d}^{4} x \sqrt{-g_{m}} \kappa R_{m}+\int \mathrm{d}^{4} x \sqrt{-g_{m}}\left(-\frac{1}{2} g_{m}^{\mu \nu} \partial_{\mu} \phi_{m} \partial_{\nu} \phi_{m}\right) .
$$

This Lagrangian and the Lagrangian for a non-minimally coupled massless scalar field,

$S_{\xi}=\frac{1}{2} \int \mathrm{d}^{4} x \sqrt{-g_{\xi}} \kappa R_{\xi}+\int \mathrm{d}^{4} x \sqrt{-g_{\xi}}\left(-\frac{1}{2} g_{\xi}^{\mu \nu} \partial_{\mu} \phi_{\xi} \partial_{\nu} \phi_{\xi}-\frac{1}{2} \xi R_{\xi} \phi_{\xi}^{2}\right)$,

can be related by a conformal transformation of the metric $g_{\mu \nu}^{\xi}=\Omega^{2} g_{\mu \nu}^{m}$ and a redefinition of the scalar field $\phi_{\xi}=\phi_{\xi}\left(\phi_{m}\right)$ [26]. Rescaling the field $\Phi=\phi / \sqrt{6 \kappa}$ we can write the specific transformation as

$$
\Omega^{2}=\frac{1}{\left(1-6 \xi \Phi_{\xi}^{2}\right)}, \quad \frac{\mathrm{d} \Phi_{m}}{\mathrm{~d} \Phi_{\xi}}= \pm \frac{\sqrt{1-6 \xi(1-6 \xi) \Phi_{\xi}^{2}}}{\left(1-6 \xi \Phi_{\xi}^{2}\right)}
$$

Note that for $0<\xi<\frac{1}{6}$ the absolute value of the non-minimally coupled scalar field cannot surpass $1 / \sqrt{6 \xi(1-6 \xi)}$ if we want expression (3.6) to make sense. For every solution of equation (3.6) we have a two-parameter family, $\{\eta, \chi\}$, of solutions for the non-minimally coupled system. For $\xi=0$, the expressions in (3.6) become the identity transformation, as they must. For $\xi=\frac{1}{6}$ we easily find the following solutions:

$$
\begin{array}{ll}
\Omega^{2}=\cosh ^{2}\left(\Phi_{m}-\Phi_{+}^{0}\right), & \Phi_{\xi=\frac{1}{6}}= \pm \tanh \left(\Phi_{m}-\Phi_{+}^{0}\right), \\
\Omega^{2}=-\sinh ^{2}\left(\Phi_{m}-\Phi_{-}^{0}\right), & \Phi_{\xi=\frac{1}{6}}= \pm \operatorname{coth}\left(\Phi_{m}-\Phi_{-}^{0}\right),
\end{array}
$$

with $\Phi_{+}^{0}$ and $\Phi_{-}^{0}$ two arbitrary real constants. The second set of solutions is unphysical because it gives a negative sign for the metric signature (opposite to the one we are using). However, one can easily demonstrate [26] that the Einstein tensor $G_{\mu \nu}$ and the energy-momentum tensor (2.2) for a massless scalar field are both invariant if we change $g_{\mu \nu}$ to $-g_{\mu \nu}$ leaving the field unchanged. Therefore, from the unphysical solutions (3.8) we obtain physical solutions of the form

$$
\Omega^{2}=\sinh ^{2}\left(\Phi_{m}-\Phi_{-}^{0}\right), \quad \Phi_{\xi=\frac{1}{6}}= \pm \operatorname{coth}\left(\Phi_{m}-\Phi_{-}^{0}\right) .
$$

We can also find additional solutions by considering the limiting cases when the constants $\Phi_{+}^{0}$ or $\Phi_{-}^{0}$ tend to $( \pm)$ infinity. In this way we find the solutions:

$$
\begin{array}{ll}
\Omega^{2}=\exp \left(2 \Phi_{m}\right), & \Phi_{\xi=\frac{1}{6}}= \pm 1, \\
\Omega^{2}=\exp \left(-2 \Phi_{m}\right), & \Phi_{\xi=\frac{1}{6}}= \pm 1 .
\end{array}
$$

All of these metrics $g_{\mu \nu}^{\left(\xi=\frac{1}{6}\right)}=\Omega^{2} g_{\mu \nu}^{m}$, described in (3.7), (3.9), (3.10) and (3.11), have a zero scalar curvature, $R=0$, owing to the conformal coupling features [15]. Therefore, any of 
these geometries, supplemented with the specific value $\Phi_{\xi}= \pm 1 / \sqrt{6 \xi}$ for the non-minimally coupled scalar field (with $\xi>0$ ) are examples of the 'trivial' solutions for the non-minimally coupled system of which we have spoken at the beginning of this section $\dagger$, and these 'trivial' solutions no longer seem to be all that trivial. These solutions cannot be obtained by means of the conformal transformation procedure described above because the conformal factor has a singular behaviour for those scalar field values.

The spacetime geometries of all these solutions were analysed in a previous paper [15] although with a slightly different parametrization. Here, we will describe them as limiting cases of the solutions that we will find for the interval $0<\xi<\frac{1}{6}$. However, we want to mention that among these conformal solutions we found traversable wormholes and that in the present parametrization they correspond to (3.7) with $\Phi_{+}^{0}>0$, (3.9) with $\Phi_{-}^{0}>0$, and (3.11); all using a value for $\chi$ in (3.2) equal to $\pi / 3$.

Let us now solve the general equation (3.6) for an arbitrary $\xi$. We first rewrite this equation as a sum of two terms

$$
\frac{\mathrm{d} \Phi_{m}}{\mathrm{~d} \Phi_{\xi}}= \pm\left[\frac{6 \xi}{\left(1-6 \xi \Phi_{\xi}^{2}\right) \sqrt{1-6 \xi(1-6 \xi) \Phi_{\xi}^{2}}}+\frac{(1-6 \xi)}{\sqrt{1-6 \xi(1-6 \xi) \Phi_{\xi}^{2}}}\right] .
$$

For convenience, we will express the solutions of this equation in terms of two functions $F\left(\Phi_{\xi}\right)$ and $H\left(\Phi_{\xi}\right)$ as

$$
\Phi_{m}\left(\Phi_{\xi}\right)= \pm \ln \left[F\left(\Phi_{\xi}\right) H\left(\Phi_{\xi}\right)\right] .
$$

The function $F$ will be related to the first term in (3.12) and the function $H$ with the second term.

\section{3. $F(\Phi)$}

The first term in (3.12) can easily be integrated by changing to a new variable

$$
v \equiv \frac{6 \xi \Phi_{\xi}}{\sqrt{1-6 \xi(1-6 \xi) \Phi_{\xi}^{2}}} .
$$

Using this new variable we only have to solve an integral of the form

$$
\int \frac{\mathrm{d} v}{1-v^{2}}
$$

that we express in terms of logarithms. In this way (once we invert the change of variables), we arrive at a closed form for the function $F$ in (3.13), for arbitrary $\xi$. Indeed, the above integral gives rise to two possible functions $F, F_{+}$and $F_{-}$, of the form

$$
F_{ \pm}\left(\Phi_{\xi}\right)=\Phi_{ \pm} \sqrt{ \pm \frac{\sqrt{1-6 \xi(1-6 \xi) \Phi_{\xi}^{2}}+6 \xi \Phi_{\xi}}{\sqrt{1-6 \xi(1-6 \xi) \Phi_{\xi}^{2}}-6 \xi \Phi_{\xi}}}
$$

for $\xi>0$, and only one, the positive sign $F_{+}$, for $\xi \leqslant 0$.

This restriction on signs is due to the fact that for $\xi>0$ the absolute value of the variable $v$ can be greater or lower than unity and these two domains have to be analysed separately. In

$\dagger$ Note that, for the conformal case, the solutions obtained by means of the limiting procedure, (3.10) and (3.11), are already in this latter class. 
terms of the scalar field, these two domains are separated by the critical value $|\Phi|=1 / \sqrt{6 \xi}$. At best, the function $F_{+}$is only defined for $|\Phi| \leqslant 1 / \sqrt{6 \xi}$, whilst $F_{-}$is at best defined for the region $|\Phi| \geqslant 1 / \sqrt{6 \xi}$. Indeed, in the case $0<\xi<\frac{1}{6}$, the function $F_{-}$is only defined up to $|\Phi| \leqslant 1 / \sqrt{6 \xi(1-6 \xi)}$.

An observation is in order at this point. In the second domain of values for the scalar field the conformal factor $\Omega^{2}=1 /\left(1-6 \xi \Phi_{\xi}^{2}\right)$ is negative and so the geometry obtained is 'unphysical' in the sense that the metric has a reversed signature. As explained before for the conformal case, from this unphysical solution we can obtain a physical solution by changing the sign of the conformal factor to $\Omega^{2}=1 /\left(6 \xi \Phi_{\xi}^{2}-1\right)$ [26]. Another observation concerning the functions $F_{ \pm}$is that we have already embedded into them the corresponding integration constants for each solution. The $\Phi_{ \pm}$in (3.16) are these integration constants. Owing to the logarithmic form in which we have cast the solution these constants are both positive. In summary,

(a) $\xi<0$ :

$F_{+}(\Phi)$ is real and well defined for all values of $\Phi$;

$F_{-}(\Phi)$ is undefined (complex), and not needed.

(b) $\xi=0$ :

$F_{+}(\Phi)=\Phi_{+}$

$F_{-}(\Phi)$ is undefined (complex), and not needed.

(c) $0<\xi<\frac{1}{6}$ :

$F_{+}(\Phi)$ is real and well defined for $|\Phi|<1 / \sqrt{6 \xi}<1 / \sqrt{6 \xi(1-6 \xi)}$;

and is undefined outside this range.

$F_{-}(\Phi)$ is real and well defined for $1 / \sqrt{6 \xi}<|\Phi|<1 / \sqrt{6 \xi(1-6 \xi)}$;

and is undefined outside this range.

(d) $\xi=\frac{1}{6}$ :

For conformal coupling there is tremendous simplification

$$
F_{ \pm}(\Phi)=\Phi_{ \pm} \sqrt{ \pm \frac{1+\Phi}{1-\Phi}} .
$$

$F_{+}(\Phi)$ is well defined for $|\Phi|<1$, whereas $F_{-}(\Phi)$ is well defined for $|\Phi|>1$.

(e) $\xi>\frac{1}{6}$ :

$F_{+}(\Phi)$ is real and well defined for $|\Phi|<1 / \sqrt{6 \xi}$;

and is undefined outside this range.

$F_{-}(\Phi)$ is real and well defined for $|\Phi|>1 / \sqrt{6 \xi}$;

and is undefined outside this range.

\section{4. $H(\Phi)$}

The second term in (3.12) can be integrated directly yielding different rather complex algebraic expressions for the function $H\left(\Phi_{\xi}\right)$ depending on the value of $\xi$ :

(a) $\xi<0$ :

$$
H\left(\Phi_{\xi}\right)=\left(\sqrt{6 \xi(6 \xi-1)} \Phi_{\xi}+\sqrt{1-6 \xi(1-6 \xi) \Phi_{\xi}^{2}}\right)^{\sqrt{(6 \xi-1) / 6 \xi}} .
$$

(b) $\xi=0$ :

$$
H\left(\Phi_{\xi}\right)=\exp \left(\Phi_{\xi}\right) .
$$


(c) $0<\xi<\frac{1}{6}$ :

$$
H\left(\Phi_{\xi}\right)=\exp \left(\sqrt{\frac{1-6 \xi}{6 \xi}} \sin ^{-1}\left(\sqrt{6 \xi(1-6 \xi)} \Phi_{\xi}\right)\right) .
$$

(d) $\xi=\frac{1}{6}$ :

$$
H\left(\Phi_{\xi}\right)=1 .
$$

(e) $\xi>\frac{1}{6}$ :

$$
H\left(\Phi_{\xi}\right)=\left(\sqrt{6 \xi(6 \xi-1)} \Phi_{\xi}+\sqrt{1-6 \xi(1-6 \xi) \Phi_{\xi}^{2}}\right)^{-\sqrt{(6 \xi-1) / 6 \xi}} .
$$

Here, we have not introduced any arbitrary integration constants because, as we mentioned before, we have already included these constants in the $F_{ \pm}$functions.

\subsection{The general solution}

At this point we already have (implicitly) all the different classical solutions for gravity plus a massless non-minimal scalar field. On one hand, if we substitute the different $F\left(\Phi_{\xi}\right)$ and $H\left(\Phi_{\xi}\right)$ in the left-hand side of (3.13), and the minimally coupled scalar field (3.2) in the right-hand side, we have an implicit expression for the non-minimally coupled scalar field as a function of the radial coordinate

$$
\left(1-\frac{2 \eta}{r}\right)^{\sin \chi / 2 \sqrt{3}}=\left[F\left(\Phi_{\xi}\right) H\left(\Phi_{\xi}\right)\right]^{ \pm 1} .
$$

These relations cannot be analytically inverted, in general. Also, depending on whether the function $F$ is $F_{+}$or $F_{-}$we have geometries $g_{\mu \nu}^{\xi}=\Omega^{2} g_{\mu \nu}^{m}$ with different conformal factors:

$$
F_{+} \rightarrow \Omega^{2}=\frac{1}{1-6 \xi \Phi_{\xi}^{2}} ; \quad F_{-} \rightarrow \Omega^{2}=\frac{1}{6 \xi \Phi_{\xi}^{2}-1} .
$$

Our next step is to analyse the different solutions found. Let us begin with some general comments. For convenience, henceforth we will work in isotropic coordinates,

$$
r=\bar{r}\left(1+\frac{\eta}{2 \bar{r}}\right)^{2}
$$

In these coordinates the previous implicit expression for the scalar field (3.22) becomes

$$
\left[\frac{1-\eta / 2 \bar{r}}{1+\eta / 2 \bar{r}}\right]^{\sin x / \sqrt{3}}=\left[F\left(\Phi_{\xi}\right) H\left(\Phi_{\xi}\right)\right]^{ \pm 1} .
$$

As in the minimally coupled case, the non-minimal solutions possess a symmetry under $(\eta, \chi)$ going to $(-\eta, \chi+\pi)$. Also, they exhibit a symmetry under the change of $\chi$ to $-\chi$ and a simultaneous flip in the sign of the exponent on the right-hand side of (3.25). Therefore, we will without loss of generality restrict the analysis to $\eta \geqslant 0, \chi \in[0, \pi]$ and the positive exponent, remembering that for every solution there is a second solution that is geometrically identical but with a reversed sign for the scalar field. 


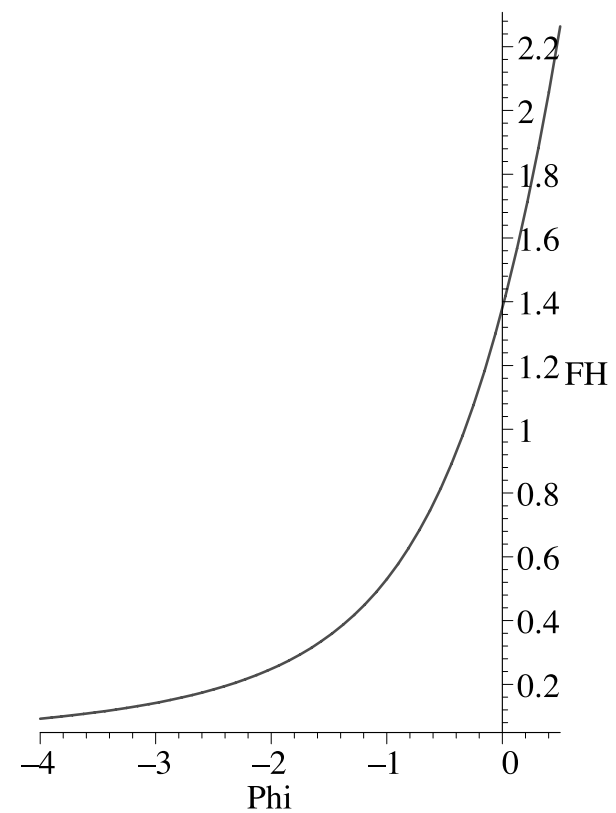

Figure 1. $F_{+} H, \xi=-0.2, \chi=\pi / 6$. There are no traversable wormholes for $\xi<0$, and the solutions generically contain naked singularities.

After a little algebra we can express the Schwarzschild radial coordinate $\mathcal{R}$ for the different geometries as a function of $\Phi_{\xi}$ and, therefore, implicitly as a function of the isotropic radial coordinate $\bar{r}$,

$$
\mathcal{R}_{ \pm}\left(\Phi_{\xi}\right)=2 \eta \frac{\left(F_{ \pm} H\right)^{\sqrt{3}(1-\cos \chi) / \sin \chi}}{\left[1-\left(F_{ \pm} H\right)^{2 \sqrt{3} / \sin \chi}\right] \sqrt{ \pm\left(1-6 \xi \Phi_{\xi}^{2}\right)}} .
$$

In the following discussion it is useful to know that, in the case $\xi>0$, if we make a perturbative expansion of the functions $F_{ \pm} H$ around $\Phi_{\xi}=-1 / \sqrt{6 \xi}$ (that is, we take

$$
\Phi_{\xi}=\frac{-1}{\sqrt{6 \xi}} \pm \epsilon,
$$

with $\epsilon$ and small positive quantity) then the functions $F_{ \pm} H \rightarrow 0$ as $\sqrt{\epsilon}$. In the same way, the conformal factor $\Omega \rightarrow \infty$ as $1 / \sqrt{\epsilon}$.

\subsection{Solutions with $\xi<0$}

In this case, the function $F_{+} H$ is everywhere positive (see figure 1). For a certain (finite) value of the scalar field (depending on $\Phi_{+}$and $\xi$ ), $F_{+} H \rightarrow 1$. We can easily verify that both $\bar{r}$ and $\mathcal{R}_{+}$go to infinity at this stage, so they are describing an asymptotic region. Indeed, it is an asymptotically flat region because since the scalar field goes to a finite constant in the asymptotic region with $\bar{r} \rightarrow \infty$ the conformal factor tends to a constant, and therefore the geometry behaves as in the minimal solution metric (3.2).

Decreasing the value of the scalar field we drive ourselves towards the interior of the geometry. As $\Phi_{\xi} \rightarrow-\infty, F_{+} H \rightarrow 0$ and $\bar{r} \rightarrow \eta / 2$. Then, analysing the asymptotic behaviour of $\Phi_{\xi}$ in equation (3.26) we can conclude that the Schwarzschild radial coordinate 


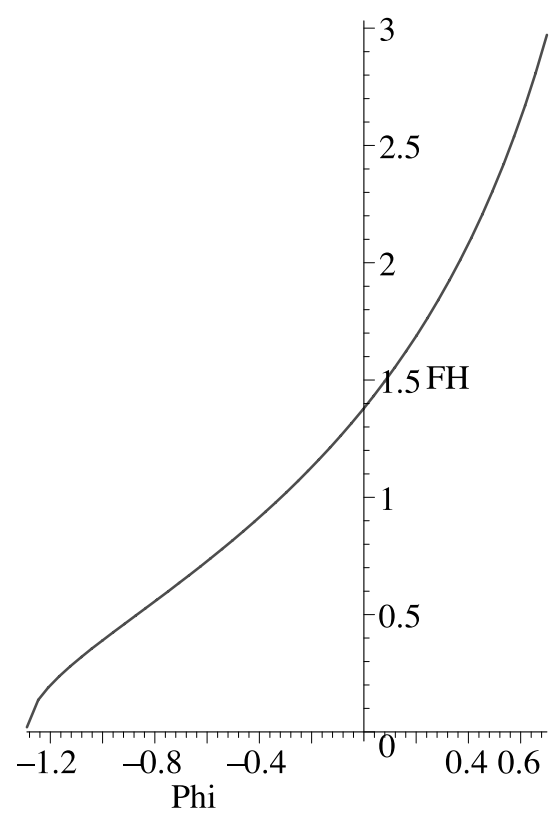

Figure 2. $F_{+} H, \xi=0.1, \chi=\pi / 6$. For $\xi \in\left(0, \frac{1}{6}\right)$ both the $F_{+}$and $F_{-}$branches need to be considered.

shrinks to zero for every $\chi \neq 0$. Thus, for a non-minimally coupled scalar field with a curvature coupling $\xi<0$ we find naked singularities, in the same manner as for a minimally coupled scalar field [30]. For the special case $\chi=0$ we find solutions with a constant value for the scalar field and a Schwarzschild geometry (of course, in this case the spacetime geometry does not shrink to zero for $\bar{r}=\eta / 2$ ), while for $\chi=\pi$ we encounter an anti-Schwarzschild geometry.

\subsection{Solutions with $\xi=0$}

The function $F(\Phi)$ in this case becomes a constant. The function $H(\Phi)$ is $\exp \left(\Phi_{\xi}\right)$, so in equation (3.13) we can read that $\Phi_{m}=\Phi_{\xi}+$ constant. Clearly, we recover the standard minimally coupled solutions with their naked singularities.

\subsection{Solutions with $0<\xi<\frac{1}{6}$}

Here we have to analyse separately both signs in equation (3.26). Let us begin with the positive, $F_{+}$. As before, for a certain value of the scalar field the function $F_{+} H \rightarrow 1$, making both $\mathcal{R}_{+} \rightarrow \infty$ and $\bar{r} \rightarrow \infty$, thereby describing an asymptotically flat region (see figure 2 ). Then, decreasing the value of the field we leave the asymptotic region going towards the interior of the geometry. At the value $-1 / \sqrt{6 \xi}$ the $\bar{r}$ coordinate reaches the value $\eta / 2$ with the corresponding zero value for $F_{+} H$. A perturbative analysis around $-1 / \sqrt{6 \xi}$ tells us that for $\chi \in(0, \pi / 3)$ the Schwarzschild radial coordinate blows up. For the rest of values the geometry shrinks to a naked singularity except for $\chi=0$ and $\pi / 3$ for which the Schwarzschild radial coordinate goes to a constant value.

The solution with $\chi=0$ is once again the Schwarzschild geometry, whilst among the naked singularities we have the $\chi=-\pi$ case representing the anti-Schwarzschild geometry. 


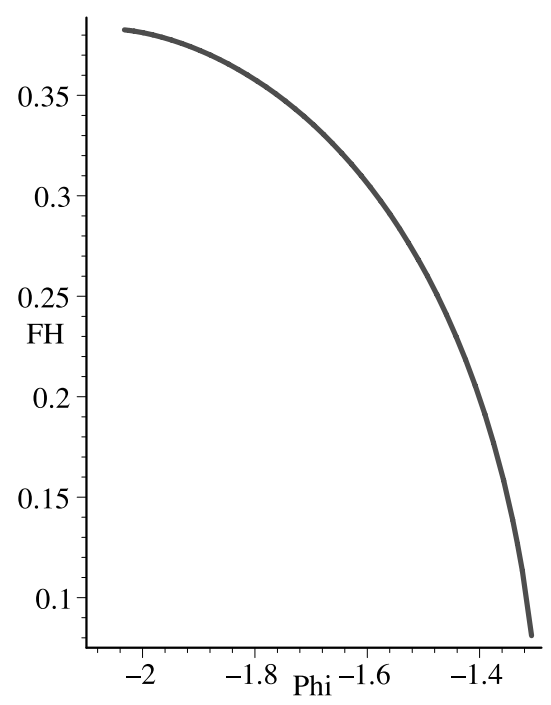

Figure 3. $F_{-} H, \xi=0.1, \chi=\pi / 6$. For $\xi \in\left(0, \frac{1}{6}\right)$ both the $F_{+}$and $F_{-}$branches need to be considered.

The solutions with $\chi=\pi / 3$ are more bizarre. At $\bar{r}=\eta / 2$ the geometry neither shrinks to zero nor blows up to infinity. We can see the reason for this behaviour easily by looking at equation (3.26). For $\chi=\pi / 3$ the exponent of the function $F_{+} H$ becomes unity and so it goes to zero at the same rate as the factor of $\sqrt{1-6 \xi \Phi_{\xi}^{2}}$, with these two terms counteracting each other. Moreover, it can be seen that $g_{t t}>0$ for $\bar{r} \geqslant \eta / 2$, that is, we do not find any horizon by going inward from the asymptotic region. In fact, we can extend these geometries to values $\bar{r}<\eta / 2$ but we will leave it for the next section to describe the traversable wormhole nature of these solutions.

The solutions with $\chi \in(0, \pi / 3)$ also deserve some additional attention. They are wormhole-like shaped, that is, they have two asymptotic regions (at $\bar{r}=\infty$ and $\bar{r}=\eta / 2$ ) joined by a throat. However, analysing the $t t$ component of the Ricci tensor $\left(R_{\hat{t} \hat{t}}\right.$ in an orthonormal coordinate basis) one can easily realize that it diverges when the scalar field reaches the value $-1 / \sqrt{6 \xi}$, that is, at $\bar{r}=\eta / 2$. Therefore, the region $\bar{r} \rightarrow \eta / 2$ is not a proper 'asymptotic' region (see the discussion on this point in [15]). Although in these geometries there exist diverging-lens effects (they have a throat), they are not genuine traversable wormholes.

The solutions with $F_{-}$in equation (3.26) merit a discussion similar to that made for the plus sign. The real function $F_{-} H$ is only defined for absolute values of the scalar field greater than $1 / \sqrt{6 \xi}$, and of course less than $1 / \sqrt{6 \xi(1-6 \xi)}$ (see figure 3 ). When the scalar field reaches a value slightly lower than $-1 / \sqrt{6 \xi}$ the radial coordinate $\bar{r}$ approaches $\eta / 2$, with $F_{-} H$ going to zero. At this coordinate point we can perform the same analysis as before, concerning the different behaviour as a function of $\chi$ of the Schwarzschild radial coordinate $\mathcal{R}_{-}$. Obviously, we find the same results. The case in which we are most interested is that of $\chi=\pi / 3$. In the next section we will see how by extending the geometry beyond $\eta / 2$ we finally obtain a perfectly well defined traversable wormhole. However, for this to happen it is necessary that by decreasing the value of the scalar field from the critical value $-1 / \sqrt{6 \xi}$, one must reach an asymptotic region before arriving at the lowest possible value of $-1 / \sqrt{6 \xi(1-6 \xi)}$. That can 
only be guaranteed if the condition

$$
\Phi_{-}>\exp \left(-\sqrt{\frac{1-6 \xi}{6 \xi}} \frac{\pi}{2}\right)
$$

is fulfilled. This condition is obtained by requiring $F_{-} H$ to have a value greater than one for $\Phi=-1 / \sqrt{6 \xi(1-6 \xi)}$. In the example of figure 3 this is not satisfied. This constraint implies super-Planckian values of the scalar field in the wormhole throat, and is a cause for some mild concern —we shall return to this point shortly.

\subsection{Solutions with $\xi=\frac{1}{6}$}

The coupling constant $\xi=\frac{1}{6}$ corresponds to a conformal coupling prescription. We have already found all the classical solutions for this system in [15], highlighting the many interesting characteristics possessed by the conformal coupling. Here, we present these solutions as a particular case of the curvature coupling. For this particular case the function $H$ is unity and the functions $F_{ \pm}$become

$$
F_{ \pm}\left(\Phi_{\left[\xi=\frac{1}{6}\right]}\right)=\Phi_{ \pm} \sqrt{ \pm \frac{1+\Phi_{\left[\xi=\frac{1}{6}\right]}}{1-\Phi_{\left[\xi=\frac{1}{6}\right]}}} .
$$

The expression (3.13) can be inverted $\dagger$ yielding the two solutions (3.7) and (3.9), provided we identify $\Phi_{-}^{0}=\ln \Phi_{-}$and $\Phi_{+}^{0}=\ln \Phi_{+}$.

The analysis done for the previous $0<\xi<\frac{1}{6}$ case extends directly to $\xi=\frac{1}{6}$. The special geometry corresponding to $\chi=\pi / 3$ will be described more fully in the next section in combination with the equivalents for the range $0<\xi<\frac{1}{6}$.

\subsection{Solutions with $\xi>\frac{1}{6}$}

Once more it is necessary to take into account the functions $F_{+}$and $F_{-}$separately. The form of $F_{+} H$ tells us that the scalar field reaches some finite value in an asymptotic region, at the point with $F_{+} H=1$ (see figure 4 ). Then, by going inward from this asymptotically flat region we approach the $\bar{r}=\eta / 2$ section, for a value of the scalar field equal to $-1 / \sqrt{6 \xi}$. Perturbatively analysing the form of $F_{+} H$ around $\Phi_{\xi}=-1 / \sqrt{6 \xi}$ we can see that $\mathcal{R}_{+}$has a different behaviour depending on the value of $\chi$. One again obtains the same qualitative results as for the previous $0<\xi<\frac{1}{6}$ case. For $0<\chi<\pi / 3$ the geometry blows up in a singular way, for $\pi / 3<\chi<\pi$ the geometry shrinks to a naked singularity, and in the special $\chi=\pi / 3$ case the geometry can be extended to values $\bar{r}<\eta / 2$. Once more, this particular case will be described in the next section.

If we now consider the function $F_{-} H$, we can see that it is only defined for $\left|\Phi_{\xi}\right|>1 / \sqrt{6 \xi}$. Here, for a negative value of the field lower than $-1 / \sqrt{6 \xi}$ we have an asymptotically flat region, $\bar{r} \rightarrow \infty\left(F_{-} H \rightarrow 1\right)$, but now this happens independently of the value of $\Phi_{-}$(see figure 5). Increasing the value of the scalar field up to $-1 / \sqrt{6 \xi}$ we arrive at the inner $\bar{r}=\eta / 2$ region. At this coordinate point we find once more what we now see are the three possible standard behaviours for the geometry depending on $\chi$.

$\dagger$ Tip: remember that $\frac{1}{2} \ln \frac{1+x}{1-x}=\tanh ^{-1}(x)$. 


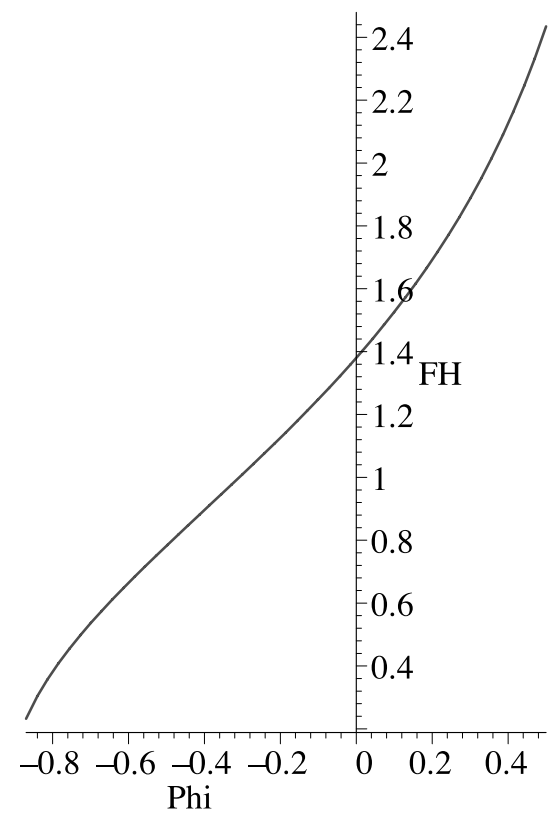

Figure 4. $F_{+} H, \xi=0.2, \chi=\pi / 6$. For $\xi \in\left(0, \frac{1}{6}\right)$ both the $F_{+}$and $F_{-}$branches need to be considered.

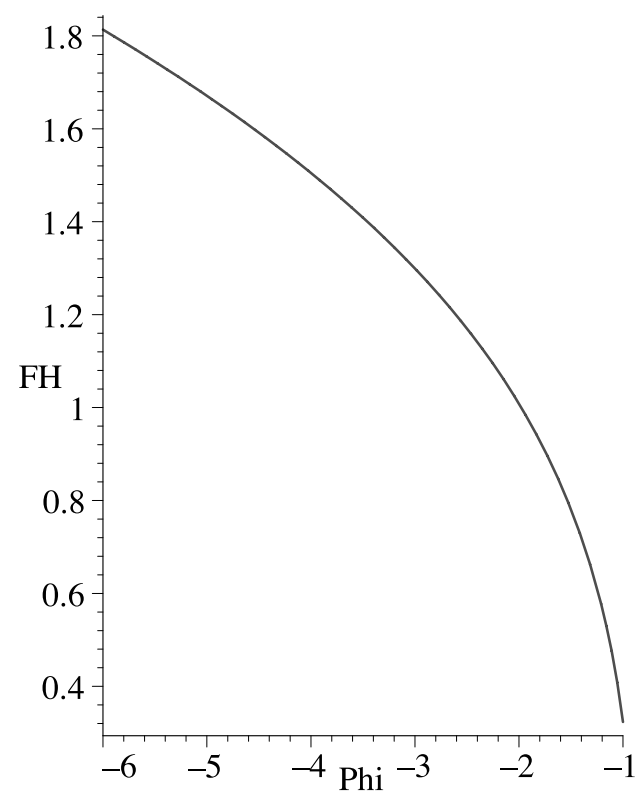

Figure 5. $F_{-} H, \xi=0.2, \chi=\pi / 6$. For $\xi \in\left(0, \frac{1}{6}\right)$ both the $F_{+}$and $F_{-}$branches need to be considered.

\section{Non-minimal scalars and traversable wormholes}

In this section we shall describe in more detail the solutions with $\chi=\pi / 3$ and $\xi>0$. In all these cases the expression under the square root symbol of the functions $F_{+}$or $F_{-}$changes its 
sign when $\Phi_{\xi}=-1 / \sqrt{6 \xi}(\bar{r}=\eta / 2)$. It seems that we cannot define the scalar field beyond this point because of the apparently complex values that $F$ would generate. However, we have to realize that for $\chi=\pi / 3$ the expression (3.25) can be written as

$$
\left[\frac{1-\eta / 2 \bar{r}}{1+\eta / 2 \bar{r}}\right]=\left[F\left(\Phi_{\xi}\right) H\left(\Phi_{\xi}\right)\right]^{2}
$$

which we shall see allows us to avoid the problem and permits the extension. By using isotropic coordinates and particularizing to the value $\chi=\pi / 3$ one can realize that there is a convenient way in which we can write the metric:

$$
\mathrm{d} s_{\xi}^{2}= \pm \frac{\left(F_{ \pm} H\right)^{2}}{1-6 \xi \Phi_{\xi}^{2}}\left[-\mathrm{d} t^{2}+\left(1+\frac{\eta}{2 \bar{r}}\right)^{4}\left[\mathrm{~d} \bar{r}^{2}+\bar{r}^{2}\left(\mathrm{~d} \theta^{2}+\sin ^{2} \theta \mathrm{d} \Phi^{2}\right)\right]\right]
$$

Let us see what happens in the $0<\xi<\frac{1}{6}$ case when we choose the function $F_{+}$. As we explained in the previous section, the implicit relation (4.1), and the form of the Schwarzschild radial coordinate (3.26), tell us that we have a geometry that is perfectly regular from $\bar{r} \rightarrow \infty$ to $\bar{r}=\eta / 2$, at which point the scalar field acquires the value $-1 / \sqrt{6 \xi}$. The function $\left(F_{+} H\right)^{2}$ has a zero for $\Phi_{\xi}=-1 / \sqrt{6 \xi}$ which counteracts the zero in the denominator of the prefactor in equation (4.2). Therefore, despite naive appearances, this prefactor acquires a finite positive value on $\Phi_{\xi}=-1 / \sqrt{6 \xi}$. Beyond that point, that is for $\bar{r}<\eta / 2$ and $\Phi<-1 / \sqrt{6 \xi}$, we can see that this prefactor continues to be finite and positive $\left(\left(F_{+} H\right)^{2}\right.$ changes its sign in the same way as $\left.\left(1-6 \xi \Phi_{\xi}^{2}\right)\right)$ even at the lowest possible value for the scalar field $-1 / \sqrt{6 \xi(1-6 \xi)}$. Now, if the radial coordinate $\bar{r}$ reaches the value zero before the scalar field reaches its lowest possible value $-1 / \sqrt{6 \xi(1-6 \xi)}$, that is, if the condition

$$
\Phi_{+}>\exp \left(-\sqrt{\frac{1-6 \xi}{6 \xi}} \frac{\pi}{2}\right)
$$

is fulfilled, expression (4.2) tells us that for $\bar{r}=0$ there is another asymptotically flat region. This spacetime is now a perfectly well defined traversable wormhole geometry.

If the condition (4.3) is not satisfied, then the scalar field reaches the critical value $-1 / \sqrt{6 \xi(1-6 \xi)}$ before the function $\left(F_{+} H\right)^{2}$ reaches the value -1 for which the other asymptotic region shows up. In these solutions, we can extend the geometry and the scalar field regularly up to a spherical section at which the scalar field approaches the value $-1 / \sqrt{6 \xi(1-6 \xi)}$. At this value we can see, by differentiating (3.25) with respect to $\bar{r}$, that the derivative of the scalar field becomes infinite. This, and the form of the scalar curvature for these systems,

$$
R_{\xi}=\frac{6\left(\nabla \Phi_{\xi}\right)^{2}(1-6 \xi)}{1-6 \xi(1-6 \xi) \Phi_{\xi}^{2}}
$$

tells us that there is a curvature singularity in these solutions. (This will be a naked singularity hiding behind a wormhole throat, but the lack of a second asymptotic region implies these are not true traversable wormhole solutions.)

We can now realize that by choosing the $F_{-}$branch and extending upwards from scalar field values greater than $-1 / \sqrt{6 \xi(1-6 \xi)}$ towards $\Phi_{\xi}>-1 / \sqrt{6 \xi}$, we obtain the same solutions as for $F_{+}$, but with interchanged asymptotic regions. The prefactor in equation (4.2) is $\left(F_{-} H\right)^{2} /\left(6 \xi \Phi_{\xi}^{2}-1\right)$ in this case. The condition (3.28) now plays the same role here as that of (4.3) previously.

The traversable wormhole solutions that we found in [15] for the conformal case can be rediscovered here as the limiting case of those with $0<\xi<\frac{1}{6}$. The conditions (3.28) and 
(4.3) for the existence of wormholes, become $\Phi_{-}>1$ and $\Phi_{+}>1$ which yield easily the conditions $\Phi_{-}^{0}=\ln \Phi_{-}>0$ and $\Phi_{+}^{0}=\ln \Phi_{+}>0$ for the conformal wormholes.

In the case $\xi>\frac{1}{6}$, and using the function $F_{+}$, we had solutions with an asymptotic region in which the scalar field acquired a value greater than $-1 / \sqrt{6 \xi}$. From this asymptotic region one can move in the direction in which the scalar field decreases. In this way, first one crosses the non-singular section with $\Phi_{\xi}=-1 / \sqrt{6 \xi}$. Later on, one crosses a spherical section with a minimum diameter (a throat), and from that point on the size of the spherical sections begins to grow to reach another asymptotic region associated with some asymptotic value for the scalar field which is of course lower than $-1 / \sqrt{6 \xi}$. Here, one does not have to impose any restriction on $\Phi_{+}$in order to obtain a traversable wormhole configuration.

By choosing the function $F_{-}$we find the same solutions, but coordinatized in a reversed way: the asymptotic region with a greater scalar field value is here that with $\bar{r}=0$.

In view of the plethora of traversable wormhole solutions we have found, an important observation is in order. In all these solutions the scalar field has to reach absolute values above $\sim m_{\mathrm{p}} / \sqrt{\xi}$, where $m_{\mathrm{p}}$ is the Planck mass. That is, either the scalar field acquires trans-Planckian values or the curvature coupling constant $\xi$ must become disturbingly large $\dagger$. Moreover, imagining that we include in the system some additional matter field with an action

$$
\mathcal{S}^{m}=\int \sqrt{-g_{\xi}} f\left(\Phi_{\xi}\right) \mathcal{L}^{m}
$$

its contribution to the effective energy-momentum tensor would be

$$
\left[T_{\mathrm{eff}}^{m}\right]_{\mu \nu}=\frac{f\left(\Phi_{\xi}\right)}{1-6 \xi \Phi_{\xi}^{2}} T_{\mu \nu}^{m} .
$$

This behaves as if this matter interacts through an 'effective Newton constant'

$$
\tilde{G}_{\text {eff }}=G_{\mathrm{N}} \frac{f\left(\Phi_{\xi}\right)}{1-6 \xi \Phi_{\xi}^{2}}=\frac{1}{8 \pi} \frac{f\left(\phi_{\xi}\right)}{\kappa-\xi \phi_{\xi}^{2}} .
$$

(This is not yet quite the physical Newton constant even in the standard case $f\left(\Phi_{\xi}\right)=1$; see section 5 below.) Then, unless $f\left(\Phi_{\xi}\right)$ is specifically chosen to counteract the $1-6 \xi \Phi_{\xi}^{2}$ factor, this effective Newton constant would change its sign from one asymptotic region to the other, producing weird effects on ordinary matterł. One can try to 'build' symmetric wormholes beginning from these asymmetric wormholes [15] and performing 'thin-shell surgery' [33]. In this way, one could potentially restrict the peculiar effects on matter to a thin region around the wormhole throat.

\section{Summary and discussion}

We have seen that a non-minimally coupled scalar field can violate all the energy conditions at a classical level. The violation, in principle, of the ANEC inspired us to look for the possible existence of traversable wormhole geometries supported by these non-minimal scalar fields. We have obtained the classical solutions for gravity plus a massless arbitrarily coupled scalar field in the restricted class of spherically symmetric and static configurations. Let us separate the different cases:

\footnotetext{
$\dagger$ This might suggest that ultimately a proper quantum treatment of these wormholes would be desirable. $\mp$ It might also be a source of problems for the stability of the solutions found, but we have not addressed this problem
} here. 
(a) $\xi<0$. We find naked singularity geometries and, in the case of a constant value for the scalar field, we recover the Schwarzschild solution.

(b) $\xi=0$. In this case we have the usual class of minimally coupled solutions with its naked singularities. Again, we recover the Schwarzschild solution for a constant field.

(c) $0<\xi<\frac{1}{6}$. We find assorted naked singularities. In some of them the geometry shrinks to zero, in others the singularity is placed in an asymptotic region, and in yet others there is a scalar curvature singularity at a finite size spherical section.

For a constant value of the field we recover the Schwarzschild solution, but if this value is exactly $\pm 1 / \sqrt{6 \xi}$ the geometry can be arbitrarily chosen from among the solutions for the conformal $\xi=\frac{1}{6}$ case.

Apart from these solutions we find a two-parameter family of perfectly well defined traversable wormhole geometries. Given an asymptotic value for the scalar field, with absolute value lower than $1 / \sqrt{6 \xi(1-6 \xi)}$, and a scalar charge, the asymptotic mass for a traversable wormhole is fixed.

(d) $\xi=\frac{1}{6}$. The case of conformal coupling leads to the same type of naked singularities that were seen before, but the scalar curvature singularities are now absent.

The different geometries can appear either in combination with a suitable spatially dependent scalar field, or with a constant field $\Phi= \pm 1$.

Here, we also have a two-parameter family of traversable wormholes with the only difference being that now the asymptotic value of the scalar field can have arbitrary values. In fact, when one asymptotic region has an infinite value for the scalar field it means that it is not asymptotically flat, degenerating to a cornucopia [15].

(e) $\xi>\frac{1}{6}$. Once again we find assorted naked singularities of the same type as in the conformal coupling.

For a special constant value of the field, $\pm 1 / \sqrt{6 \xi}$, the geometry can again be arbitrarily selected from among the solutions for the conformal $\frac{1}{6}$ case. For a different constant value we only recover the Schwarzschild solution.

Also, there are specific traversable wormhole solutions in which an infinite asymptotic value for the scalar field cannot be reached.

Since the potential existence of traversable wormholes is a perhaps somewhat disturbing possibility, we feel it a good idea to see where the potential pitfalls might be - mathematically, we have exhibited exact classical traversable wormhole solutions to the Einstein equations, and now wish to investigate the extent to which they should physically be trusted.

To start with, scalar fields play a somewhat ambiguous role in modern theoretical physics: on the one hand, they provide great toy models, and are from a theoretician's perspective almost inevitable components of any reasonable model of empirical reality; on the other hand, the direct experimental/observational evidence is patchy.

The only scalar fields for which we have really direct 'hands-on' experimental evidence are the scalar mesons (pions $\pi$; kaons $K$; and their 'charm', 'truth' and 'beauty' relatives, plus a whole slew of resonances such as the $\left.\eta, f_{0}, \eta^{\prime}, a_{0}, \ldots\right)$ [34]. Not a single one of these particles are fundamental, they are all quark-antiquark bound states, and while the description in terms of scalar fields is useful when these systems are probed at low momenta (as measured in their rest frame) we should certainly not continue to use the scalar field description once the system is probed with momenta greater than $\hbar /$ (bound state radius). In terms of the scalar field itself, this means you should not trust the scalar field description if gradients become large, if

$$
\|\nabla \phi\|>\frac{\|\phi\|}{\text { bound state radius }}
$$


Similarly, you should not trust the scalar field description if the energy density in the scalar field exceeds the critical density for the quark-hadron phase transition. (Note that if the scalar mesons were strict Goldstone bosons (exactly massless) rather than pseudo-Goldstone bosons, then they could achieve arbitrarily large values of the field variable with zero energy cost.) Thus scalar mesons are a mixed bag: they definitely exist, and we know quite a bit about their properties, but there are stringent limitations on how far we should trust the scalar field description.

The next candidate scalar field that is closest to experimental verification is the Higgs particle responsible for electroweak symmetry breaking. While in the standard model the Higgs is fundamental, and while almost everyone is firmly convinced that some Higgs-like scalar field exists, there is a possibility that the physical Higgs (like the scalar mesons) might itself be a bound state of some deeper level of elementary particles (e.g. technicolour and its variants). Despite the tremendous successes of the standard model of particle physics we do not (currently) have direct proof of the existence of a fundamental Higgs scalar field.

Accepting for now the existence of a fundamental Higgs scalar, what is its curvature coupling? The parameter $\xi$ is completely unconstrained in the flat-space standard model. If we choose for technical reasons to adopt the 'new improved stress-energy tensor' for the Higgs scalar in flat spacetime then one is naturally led to conformal coupling in curved spacetime [15]. Conformal coupling seems to be the 'most natural' choice for the Higgs curvature coupling, and we have seen in this paper that both conformal coupling and the entire open half-line $\xi \in(0, \infty)$ surrounding conformal coupling lead to traversable wormhole geometries. Unfortunately, adding a Higgs mass results in analytically intractable equations.

A third candidate scalar field of great phenomenological interest is the axion: it is extremely difficult to see how one could make strong interaction physics compatible with the observed lack of strong CP violation, without something like an axion to solve the so-called 'strong CP problem'. Still, the axion has not yet been directly observed experimentally.

A fourth candidate scalar field of phenomenological interest specifically within the astrophysics/cosmology community is the so-called 'inflaton'. This scalar field is used as a mechanism for driving the anomalously fast expansion of the universe during the inflationary era. While observationally it is a relatively secure bet that something like cosmological inflation (in the sense of anomalously fast cosmological expansion) actually took place, and while scalar fields of some type are presently viewed as the most reasonable way of driving inflation, we must again admit that direct observational verification of the existence of the inflaton field (and its variants, such as quintessence) is far from being accomplished. Note that in many forms of inflation trans-Planckian values of the scalar field are generic and widely accepted (though not universally accepted) as part of the inflationary paradigm.

A fifth candidate scalar field of phenomenological interest specifically within the general relativity community is the so-called 'Brans-Dicke scalar'. This is perhaps the simplest extension to Einstein gravity that is not ruled out by experiment. (It is certainly greatly constrained by observation and experiment, and there is no positive experimental data guaranteeing its existence, but it is not ruled out.) The relativity community views the Brans-Dicke scalar mainly as an excellent testing ground for alternative ideas and as a useful way of parametrizing possible deviations from Einstein gravity. (And experimentally and observationally, Einstein gravity still wins.)

In this regard it is important to emphasize that the type of scalar fields we have been discussing in the present paper are completely compatible with current experimental limits on Brans-Dicke scalars, or more generally, generic scalar-tensor theories [35,36]. To take the observational limits and utilize them in our formalism you need to use the translations

$$
\Phi_{\text {Brans-Dicke }}=\kappa-\xi \phi_{\xi}^{2},
$$




$$
\omega\left(\Phi_{\text {Brans-Dicke }}\right)=\frac{\Phi_{\text {Brans-Dicke }}}{\left(\mathrm{d} \Phi_{\text {Brans-Dicke }} / \mathrm{d} \phi_{\xi}\right)^{2}}=\frac{\kappa-\xi \phi_{\xi}^{2}}{4 \xi^{2} \phi_{\xi}^{2}} .
$$

Then, the physical Newton constant, which takes its meaning within the perturbative PPN expansion around flat Minkowski space, can be written as

$G_{\text {physical }}=\left.\frac{1}{\Phi_{\text {Brans-Dicke }}}\left(\frac{4+2 \omega}{3+2 \omega}\right)\right|_{\text {asymp }}=\left.\frac{1}{8 \pi} \frac{1}{\kappa-\xi \phi_{\xi}^{2}}\left(\frac{4+2 \omega}{3+2 \omega}\right)\right|_{\text {asymp }}$.

(Here, we have set the function $f\left(\phi_{\xi}\right)$ in (4.7) equal to one. However, it must be pointed out that if $f\left(\phi_{\xi}\right) \neq 1$ then the current theories are more general even than the standard scalartensor models.) In the wormhole solutions that we have found, the scalar field $\phi_{\xi}$ reaches a trans-Planckian value in one of the asymptotic regions, making the physical Newton constant negative. This means that, contrary to what is commonly done in scalar-tensor theories [37], the translated Brans-Dicke field should not be restricted to only positive values. In order to cover our wormhole solutions, negative values of the Brans-Dicke field are required.

Current solar system measurements imply $\left\|\omega\left(\Phi_{\text {Brans-Dicke }}\right)\right\|>3000$ [38]. More precisely, VLBI techniques allow us to use the deflection of light by the Sun to place very strong constraints on the PPN parameter $\gamma$, with [38]

$$
\gamma=1-(0.6 \pm 3.1) \times 10^{-4} \text {. }
$$

The standard result that for Brans-Dicke theories $[35,36]$

$$
\gamma=\frac{\omega+1}{\omega+2}
$$

now provides the limit on $\|\omega\|$ quoted above. For instance, this constraint is very easily satisfied if the scalar field $\phi_{\xi}$ is a small fraction of the naive Planck scale $(\sqrt{\kappa})$ in the solar neighbourhood. Thus, the scalar theories we investigate in this paper are perfectly compatible with known physics.

Finally, the membrane-inspired field theories (low-energy limits of what used to be called string theory) are literally infested with scalar fields. In membrane theories it is impossible to avoid scalar fields, with the most ubiquitous being the so-called 'dilaton'. However, the dilaton field is far from unique, in general, there is a large class of so-called 'moduli' fields, which are scalar fields corresponding to the directions in which the background spacetime geometry is particularly 'soft' and easily deformed. So if membrane theory really is the fundamental theory of quantum gravity, then the existence of fundamental scalar fields is automatic, with the field theory description of these fundamental scalars being valid at least up to the Planck scale and possibly higher.

(For good measure, by making a conformal transformation of the spacetime geometry it is typically possible to put membrane-inspired scalar fields into a framework which closely parallels that of the generalized Brans-Dicke fields. Thus there is a potential for much cross-pollination between Brans-Dicke-inspired variants of general relativity and membraneinspired field theories.)

So overall, we have excellent theoretical reasons to expect that scalar field theories are an integral part of reality, but the direct experimental/observational verification of the existence of fundamental fields is still an open question. Nevertheless, we think it fair to say that there are excellent reasons for taking scalar fields seriously, and excellent reasons for thinking that the gravitational properties of scalar fields are of interest cosmologically, astrophysically and for providing fundamental probes of general relativity. The fact that scalar fields then lead to such widespread violations of the energy conditions [14-16,23], with potentially far-reaching 
consequences like a 'universal bounce' (instead of a big-bang singularity) [23,39, 40], the traversable wormholes of this paper (see also $[15,16]$ ), and possibly even weirder physics (see, for example, [2]), leads to a rather sobering assessment of the marked limitations of our current understanding.

\section{Acknowledgments}

The research of CB was supported by the Spanish Ministry of Education and Culture (MEC). MV was supported by the US Department of Energy. MV wishes to thank Jacob Bekenstein for his interest and comments.

\section{References}

[1] Hawking S W and Ellis GF R 1973 The Large Scale Structure of Space-Time (Cambridge: Cambridge University Press)

[2] Visser M 1995 Lorentzian Wormholes: from Einstein to Hawking (New York: American Institute of Physics)

[3] Schoen R and Yau S T 1979 Commun. Math. Phys. 6545

[4] Olum K D 1998 Phys. Rev. Lett. 813567

(Olum K D 1998 Preprint gr-qc/9805003)

[5] Visser M, Bassett B and Liberati S 2000 Proc. 3rd meeting on Constrained Dynamics and Quantum Gravity QG99(Villasimius, 1999) Nucl. Phys. Proc. Suppl. B 88267

(Visser M, Bassett B and Liberati S 1998 Superluminal censorship Preprint gr-qc/9810026)

[6] Visser M, Bassett B and Liberati S 1999 Perturbative superluminal censorship and the null energy condition Proc. 8th Canadian Conf. on General Relativity and Relativistic Astrophysics ed C P Burgess and R C Meyers (New York: AIP) pp 301-5

(Visser M, Bassett B and Liberati S 1999 Preprint gr-qc/990802)

[7] Friedman J L, Schleich K and Witt D M 1993 Phys. Rev. Lett. 711486

[8] Mayo A E and Bekenstein J D 1996 Phys. Rev. D 545059

[9] Visser M 1992 Phys. Rev. D 462445

(Visser M 1992 Preprint hep-th/9203057)

[10] Senovilla J M M 1998 Gen. Rel. Grav. 30701

[11] Morris M S and Thorne K S 1988 Am. J. Phys. 56395

[12] Hochberg D and Visser M 1998 Phys. Rev. Lett. 81746

[13] Hochberg D, Popov A and Sushkov S V 1997 Phys. Rev. Lett. 782050

[14] Flanagan E E and Wald R M 1996 Phys. Rev. D 546233

[15] Barceló C and Visser M 1999 Phys. Lett. B 466127

[16] Visser M and Barceló C 2000 Energy conditions and their cosmological implications Proc. Cosmo99 (Trieste, 1999) (Singapore: World Scientific)

(Visser M and Barceló C 2000 Preprint gr-qc/0001099)

[17] Agnese A and La Camera M 1995 Phys. Rev. D 512011

[18] Nandi K K, Islam A and Evans J 1997 Phys. Rev. D 552497

[19] Anchordoqui L A, Perez Bergliaffa S and Torres D F 1997 Phys. Rev. D 555226

[20] Visser M and Hochberg D 1997 Generic wormhole throats The Internal Structure of Black Holes and Spacetime Singularities ed L M Burko and A Ori (Bristol: IOP Publishing)

(Visser M and Hochberg D 1997 Preprint gr-qc/9710001)

[21] Hochberg D 1990 Phys. Lett. B 251349

[22] Bhawal B and Kar S 1992 Phys. Rev. D 462464

[23] Bekenstein J D 1975 Phys. Rev. D 112072

[24] Visser M 1997 Science 276 88-90

[25] Froyland J 1982 Phys. Rev. D 251470

[26] Bekenstein J D 1974 Ann. Phys. 82535

[27] Duruisseau J P 1983 Gen. Rel. Grav. 15285

[28] Fisher I Z 1948 Zh. Eksp. Teor. Fiz. 18636

(Fisher I Z 1999 Preprint gr-qc/9911008)

[29] Janis A I, Newman E T and Winicour J 1968 Phys. Rev. Lett. 20878

[30] Wyman M 1981 Phys. Rev. D 24839 
[31] Cavaglia M and De V Alfaro 1997 Int. J. Mod. Phys. D 639

[32] Agnese A and La Camera M 1985 Phys. Rev. D 311280

[33] Visser M 1989 Phys. Rev. D 393182

[34] Particle Data Group 1998 Eur. Phys. J. C 31 http://pdg.lbl.gov

[35] Will C M 1993 Theory and Experiment in Gravitational Physics revised edn (Cambridge: Cambridge University Press)

[36] Weinberg S 1972 Gravitation and Cosmology (New York: Wiley)

[37] Santiago D I and Silbergleit A S 2000 Gen. Rel. Grav. 32565

(Santiago D I and Silbergleit A S 1999 On the energy momentum tensor of the scalar field in scalar-tensor theories of gravity Preprint gr-qc/9904003)

[38] Eubanks T M, Matsakis D N, Martin J O, Archinal B A, McCarthy D D, Klioner S A, Shapiro S and Shapiro I I 1997 Advances in Solar System Tests of Gravity, Proc. Joint APS/AAPT 1997 Meeting (Washington DC, 18-21 April 1997) http://flux.aps.org/meetings/YR97/BAPSAPR97/abs/G1280005.html (abstract only)

[39] Hochberg D, Molina-Paris C and Visser M 1999 Phys. Rev. D 59044011 (Hochberg D, Molina-Paris C and Visser M 1998 Preprint gr-qc/9810029)

[40] Molina-Paris C and Visser M 1999 Phys. Lett. B 455 90-5

(Molina-Paris C and Visser M 1998 Preprint gr-qc/9810023) 